\title{
Dynamics of Vortices in Chiral Media: The Chiral Propulsion Effect
}

\author{
Yuji Hirono, ${ }^{1,2}$ Dmitri E. Kharzeev, ${ }^{3,4}$ and Andrey V. Sadofyev ${ }^{5}$ \\ ${ }^{1}$ Asia Pacific Center for Theoretical Physics, Pohang 37673, Korea \\ ${ }^{2}$ Department of Physics, POSTECH, Pohang 37673, Korea \\ ${ }^{3}$ Department of Physics and Astronomy, Stony Brook University, Stony Brook, New York 11794-3800, USA \\ ${ }^{4}$ Department of Physics and RIKEN-BNL Research Center, Brookhaven National Laboratory, \\ Upton, New York 11973-5000, USA \\ ${ }^{5}$ Theoretical Division, MS B283, Los Alamos National Laboratory, Los Alamos, New Mexico 87545, USA
}

(Received 29 January 2018; published 1 October 2018)

\begin{abstract}
We study the motion of vortex filaments in chiral media and find a semiclassical analog of the anomalyinduced chiral magnetic effect. The helical solitonic excitations on vortices in a parity-breaking medium are found to carry additional energy flow along the vortex in the direction dictated by the sign of chirality imbalance; we call this new transport phenomenon the chiral propulsion effect. The dynamics of vortex filaments in the parity-breaking background is described by a modified version of the localized induction equation. We analyze the linear stability of simple vortex solutions and study the effects of chiral media on the excitation spectrum and the growth rate of the unstable modes. We also show that, if the equation of motion of the filament is symmetric under the simultaneous reversal of parity and time, planar-shape solutions cannot transport energy.
\end{abstract}

DOI: 10.1103/PhysRevLett.121.142301

Introduction.-The physics of chiral media has attracted significant attention recently. Remarkably, it is found that the quantum chiral anomaly [1,2] affects the macroscopic behavior of chiral media and induces new transport phenomena, such as the chiral magnetic [3-7] and chiral vortical effects [8-12] (CME and CVE, respectively). CME and CVE refer to the generation of electric currents along an external magnetic field or vorticity in the presence of a chirality imbalance. The resulting currents are nondissipative due to the protection by the global topology of the gauge field. These chiral effects are expected to occur in a variety of systems: the quark-gluon plasma, Dirac and Weyl semimetals, primordial electroweak plasma, and cold atoms. In quark-gluon plasma, the chirality imbalance can be produced by topological fluctuations of quantum chromodynamics, or by the combination of electric and magnetic fields that accompany heavy-ion collisions. The parallel electric and magnetic fields can also be used to create the chirality imbalance in condensed matter systems, see, e.g., Ref. [13]. In addition, CME and CVE lead to a new class of instabilities in these systems [14-20].

The CME has been observed experimentally in Dirac [13,21,22] and Weyl semimetals [23-25]. There is an

Published by the American Physical Society under the terms of the Creative Commons Attribution 4.0 International license. Further distribution of this work must maintain attribution to the author(s) and the published article's title, journal citation, and DOI. Funded by SCOAP . ongoing search for CME and the local parity violation $[3,4]$ induced by the topological fluctuations in the quarkgluon plasma in heavy-ion collisions at Relativistic HeavyIon Collider and Large Hadron Collider; see Ref. [6] for a review. In particular, the forthcoming isobar run in the Spring of 2018 at Relativistic Heavy-Ion Collider is expected to provide a conclusive result on the occurrence of CME in heavy-ion collisions [26].

Recently, the STAR collaboration reported the experimental observation of $\Lambda$ hyperon polarization along the normal to the reaction plane of the heavy-ion collision, pointing toward the existence of large vorticity in the produced quark-gluon fluid [27]. The role of vortical flows in heavy-ion collisions has been discussed, e.g., in Refs. [28-39]. It is natural to ask how the dynamics of vortices is influenced by the chiral anomaly.

In this Letter, we analyze the dynamics of vortices in a fluid with broken parity; the electromagnetic fields are treated as fully dynamical. We find a new chiral transport effect - an additional energy flow along the vortex filament in the direction determined by the sign of chirality imbalance, the chiral propulsion effect (CPE).

Motions of vortices in chirally imbalanced media.-Let us consider the motion of a vortex filament in a fluid. The vorticity concentrated on the vortex sources the velocity field according to its definition $\boldsymbol{\omega}=\nabla \times \boldsymbol{v}$, where $\boldsymbol{v}$ and $\boldsymbol{\omega}$ are fluid velocity and vorticity, respectively. The velocity field can be found analogously to the magnetic field of a thin current (Biot-Savart law). Assuming that the vortex moves on the flow and the contribution to the velocity from 
distant parts of the vortex is unimportant (localized induction approximation), the dynamics of a thin vortex filament in an incompressible and inviscid fluid is governed by the localized induction equation (LIE) [40],

$$
\dot{\boldsymbol{X}}=C \boldsymbol{X}^{\prime} \times \boldsymbol{X}^{\prime \prime},
$$

where $\boldsymbol{X}=\boldsymbol{X}(t, s)$ denotes the position of a vortex, $t$ is the time, $s$ is the arc-length parameter, the dot and prime indicate $t$ and $s$ derivatives, respectively, and $C$ is a constant proportional to the circulation. Although originally the LIE was introduced for thin vortices in classical fluids $[41,42]$, it can also describe the dynamics of quantized vortices in superfluids and superconductors [43-45]. Solving the LIE (1) is much simpler than solving the Euler equation directly, and it provides a physically intuitive picture of the dynamics of the fluid in terms of filament motions. Remarkably, the LIE (1) can be mapped to the nonlinear Schrödinger equation (NLSE) by the so-called Hasimoto transformation [46],

$$
\psi(t, s)=\kappa(t, s) \exp \left(i \int{ }^{s} \tau\left(t, s^{\prime}\right) d s^{\prime}\right),
$$

where $\kappa(t, s)$ is the curvature and $\tau(t, s)$ is the torsion of a vortex. NLSE is known to be a completely integrable system which has solitonic solutions and an infinite sequence of commuting conserved charges. The Hasimoto transformation has been shown to be a Poisson map that preserves the Poisson structures [47]. The LIE, thus, describes a completely integrable system. The solitons of NLSE correspond to the helical excitations in LIE that propagate along the vortex. They are known as Hasimoto solitons; their existence is established experimentally $[48,49]$.

Let us now consider a system in which parity is broken by the presence of chirality imbalance; the corresponding term in the action is

$$
S_{\chi}=\int d t \mu \mathcal{H},
$$

where $\mu$ is the "chiral" chemical potential (usually it is denoted by $\mu_{5}$, but as this is the only chemical potential that appears in this Letter, we simplify this notation to $\mu$. Please note that the chiral chemical potential does not correspond to a conserved quantity, as the chiral charge is not conserved due to the chiral anomaly. The state with $\mu \neq 0$, therefore, does not correspond to a true ground state of the system; for discussion, see, e.g., Ref. [50].), and $\mathcal{H}$ is the magnetic helicity given by $\mathcal{H}=\left(e^{2} / 4 \pi^{2}\right) \int d^{3} x \boldsymbol{A} \cdot \boldsymbol{B}$, where $\boldsymbol{A}$ is the vector potential and $\boldsymbol{B}$ is the magnetic field. For simplicity, we assume that the chiral chemical potential is homogenous and time independent. It is worth mentioning that taking the derivative of this action with respect to the vector potential, one readily finds the CME current: $\boldsymbol{J}_{\mathrm{CME}}=\delta S_{\chi} / \delta \boldsymbol{A} \propto \boldsymbol{B}$.
Supplementing the nonrelativistic Abelian Higgs model with Eq. (3), one can find the equation of motion for a quantized magnetic vortex at finite $\mu$, as derived by Kozhevnikov [51,52]

$$
\dot{\boldsymbol{X}}=C \boldsymbol{X}^{\prime} \times \boldsymbol{X}^{\prime \prime}+\mu\left(\boldsymbol{X}^{\prime \prime \prime}+\frac{3}{2}\left(\boldsymbol{X}^{\prime \prime}\right)^{2} \boldsymbol{X}^{\prime}\right) .
$$

The term $\mu \boldsymbol{X}^{\prime \prime \prime}$ comes from the Lorentz force exerted on the CME current along the vortex $\boldsymbol{F}=\boldsymbol{J}_{\mathrm{CME}} \times \boldsymbol{B}=$ $\mu \int_{-\infty}^{\infty} d z\left[\boldsymbol{X}^{\prime}(t, s) \times \boldsymbol{X}^{\prime}(t, s+z)\right] e^{-|z| / a}=\mu \boldsymbol{X}^{\prime} \times \boldsymbol{X}^{\prime \prime \prime}+\cdots$ where $a$ is the inverse photon mass [Eq. (4) is obtained by $\left.\boldsymbol{X}^{\prime} \times \boldsymbol{F}\right]$. The tangential term $\frac{3}{2} \mu\left(\boldsymbol{X}^{\prime \prime}\right)^{2} \boldsymbol{X}^{\prime}$ is added to keep the arc-length-preserving property (the term $\boldsymbol{X}^{\prime \prime \prime}$ can also be derived in a fluid-dynamical system using the kinetic helicity as the Hamiltonian [53]). Note that the tangential motion does not change the shape of the vortex. Hereafter, we set the constant $C$ in Eq. (4) to unity by a corresponding time rescaling. Let us note that Eq. (4) has previously emerged in a different context: it describes the motion of a vortex tube containing an axial flow and is known as the Fukumoto-Miyazaki equation (FME) [54,55]. Notably, through the Hasimoto transformation, the FME can be mapped to the integrable Hirota equation [56]

$$
i \dot{\psi}+\psi^{\prime \prime}+\frac{1}{2}|\psi|^{2} \psi-i \mu\left(\psi^{\prime \prime \prime}+\frac{3}{2}|\psi|^{2} \psi^{\prime}\right)=0 ;
$$

this map can be utilized to obtain the solitons of the FME.

Let us give some simple solutions of the FME (4). We can find a solution which has the form of a helix

$$
\boldsymbol{X}_{\text {helix }}(t, s)=\frac{1}{A^{2}}\left(\begin{array}{c}
\kappa_{0} \cos \left[A\left(s-v_{p} t\right)\right] \\
\kappa_{0} \sin \left[A\left(s-v_{p} t\right)\right] \\
\tau_{0} A\left(s-v_{g} t\right)
\end{array}\right),
$$

where the constants $\kappa_{0}$ and $\tau_{0}$ give the curvature and the torsion of the helix, $A=\sqrt{\kappa_{0}^{2}+\tau_{0}^{2}}$, and the phase and group velocities are given by $v_{p}=\tau_{0}+\mu\left[\tau_{0}^{2}-\left(\kappa_{0}^{2} / 2\right)\right]$, $v_{g}=-\kappa_{0}^{2} / \tau_{0}-\left(3 \kappa_{0}^{2} / 2\right) \mu$. Note that the sign of $\tau_{0}$ determines the handedness of the helix. The radius $R$ and the pitch $\ell$ of the helix are given by $R=\kappa_{0} /\left(\kappa_{0}^{2}+\tau_{0}^{2}\right)$, $\ell=2 \pi \tau_{0} /\left(\kappa_{0}^{2}+\tau_{0}^{2}\right)$. The solution is reduced to a circular loop in the limit $\tau_{0}=0$ in Eq. (6).

Using the map between the FME and the Hirota equation, we find a propagating solitonic solution of the FME

$$
\boldsymbol{X}_{\mathrm{sol}}(t, s)=\left(\begin{array}{c}
-\frac{2 \epsilon}{\epsilon^{2}+\tau_{0}^{2}} \operatorname{sech}[\epsilon \xi] \cos [\eta] \\
-\frac{2 \epsilon}{\epsilon^{2}+\tau_{0}^{2}} \operatorname{sech}[\epsilon \xi] \sin [\eta] \\
s-\frac{2 \epsilon}{\epsilon^{2}+\tau_{0}^{2}} \tanh [\epsilon \xi]
\end{array}\right)
$$


where $\eta \equiv \tau_{0} s+\left(\epsilon^{2}-\tau_{0}^{2}\right) t+\mu \tau_{0}\left(3 \epsilon^{2}-\tau_{0}^{2}\right), \quad \xi \equiv s-\left[2 \tau_{0}+\right.$ $\left.\mu\left(3 \tau_{0}^{2}-\epsilon^{2}\right)\right] t$, and $\epsilon$ and $\tau_{0}$ are constants. This soliton has a constant torsion given by $\tau_{0}$ and propagates in the $z$ direction. Its speed is modified by $\mu$ and reduces to the original Hasimoto soliton at $\mu=0$.

Let us discuss the kinetic properties of these solutions. The kinetic energy of a soliton can be found as $E=\frac{1}{2} \int d s \kappa^{2}=4 \epsilon$. In addition, the helical nature of the configuration [57] can be characterized by the quantity $\mathcal{H}=\int d s \kappa^{2} \tau=8 \epsilon \tau_{0}$. This is the second conserved quantity in the nonlinear Schrödinger hierarchy $[47,57]$. In the case of a planar configuration, namely if the torsion is vanishing, $\mathcal{H}=0$. Note that these quantities do not depend on $\mu$.

Let us now turn to the momentum carried by these solutions. In the thin vortex limit, the electromagnetic fields can be expressed in terms of the vortex coordinates $\boldsymbol{X}(t, s)$ as

$$
\begin{aligned}
& \boldsymbol{B}(t, \boldsymbol{x})=\varphi \int d s \boldsymbol{X}^{\prime} \delta(\boldsymbol{x}-\boldsymbol{X}), \\
& \boldsymbol{E}(t, \boldsymbol{x})=-\varphi \int d s \dot{\boldsymbol{X}} \times \boldsymbol{X}^{\prime} \delta(\boldsymbol{x}-\boldsymbol{X}),
\end{aligned}
$$

where $\varphi$ is the magnetic flux and the electric field locally has the structure " $\boldsymbol{v} \times \boldsymbol{B}$." The momentum of the magnetic flux is given by the Poynting vector

$$
\boldsymbol{P}=\int d^{3} x \boldsymbol{E} \times \boldsymbol{B}=\varphi^{2} M^{2} \int d s \dot{\boldsymbol{X}},
$$

where $M$ is the inverse of the core size of the vortex. The helix solution moves in the $z$ direction. The $z$ component of momentum per unit length of the coil is evaluated as

$$
\left(\bar{P}_{\text {helix }}\right)_{z}=\frac{\kappa_{0}^{2}}{\tau_{0}}\left(1+\frac{3}{2} \mu \tau_{0}\right) \varphi^{2} M^{2} .
$$

The $z$ component of the momentum of the soliton solution can be calculated using Eq. (7)

$$
\left(P_{\mathrm{sol}}\right)_{z}=\frac{4 \epsilon\left[2 \tau_{0}+\mu\left(3 \tau_{0}^{2}-\epsilon^{2}\right)\right]}{\epsilon^{2}+\tau_{0}^{2}} \varphi^{2} M^{2} .
$$

In both cases, there are contributions proportional to $\mu$. Therefore, the chiral medium provides a thrust to the solitons, propelling them along the vortex-we will call this the chiral propulsion effect.

In the case of the solitons (12), at $\mu=0$ the velocity is proportional to the torsion $\tau_{0}$-this means that for the excitation to have a finite momentum in a chirally symmetric medium, the vortex has to deform in a paritybreaking way. On the other hand, even if the solution is planar, it can still experience the thrust if parity is broken in the medium. Indeed, Eq. (12) shows that for $\mu \neq 0$ the thrust remains even in the $\tau_{0} \rightarrow 0$ limit corresponding to a planar solution with $\mathcal{H}=0$. As we will discuss later, a planar solution is forbidden to have a finite energy flow in a $P T$ symmetric theory. The LIE has the $P T$ symmetry, whereas in the FME case it is broken.

Properties of fluctuations. - Let us now examine the effect of the chiral medium on the fluctuations around the circle and helix solutions. We use the local coordinate system called the Frenet-Serret (FS) frame, which is commonly used to parametrize the shape of a curve. There is an ambiguity in the parametrization in $s$, and we fix this by requiring $\left|\boldsymbol{X}^{\prime}\right|=1$. Then, the unit tangent vector is written as $\boldsymbol{t}=\boldsymbol{X}^{\prime}$. Given a curvature $\kappa(t, s)$ and a torsion $\tau(t, s)$, the shape of a curve is determined, up to a trivial translation and rotation, by the FS formulas

$$
\partial_{s}\left(\begin{array}{l}
\boldsymbol{t} \\
\boldsymbol{n} \\
\boldsymbol{b}
\end{array}\right)=\left(\begin{array}{ccc}
0 & \kappa & 0 \\
-\kappa & 0 & \tau \\
0 & -\tau & 0
\end{array}\right)\left(\begin{array}{l}
\boldsymbol{t} \\
\boldsymbol{n} \\
\boldsymbol{b}
\end{array}\right),
$$

where $\boldsymbol{n} \propto \boldsymbol{t}^{\prime}$ is the unit normal vector and $\boldsymbol{b} \equiv \boldsymbol{t} \times \boldsymbol{n}$ is the unit binormal vector. The time evolution of a curve is described by

$$
\partial_{t}\left(\begin{array}{l}
\boldsymbol{t} \\
\boldsymbol{n} \\
\boldsymbol{b}
\end{array}\right)=\left(\begin{array}{ccc}
0 & \alpha & \beta \\
-\alpha & 0 & \gamma \\
-\beta & -\gamma & 0
\end{array}\right)\left(\begin{array}{l}
\boldsymbol{t} \\
\boldsymbol{n} \\
\boldsymbol{b}
\end{array}\right),
$$

where $\alpha, \beta, \gamma$ are functions of $\kappa$ and $\tau$, and their functional forms are determined from Eq. (4). The FS basis has to satisfy the compatibility conditions, $\partial_{s} \partial_{t} t=\partial_{t} \partial_{s} t$, $\partial_{s} \partial_{t} \boldsymbol{n}=\partial_{t} \partial_{s} \boldsymbol{n}, \partial_{s} \partial_{t} \boldsymbol{b}=\partial_{t} \partial_{s} \boldsymbol{b}$. Using these conditions, we find the time-evolution equations for $\tau$ and $\kappa$ [58]

$\dot{\kappa}=-2 \tau \kappa^{\prime}-\kappa \tau^{\prime}+\frac{\mu}{2}\left(2 \kappa^{\prime \prime \prime}-6 \tau^{2} \kappa^{\prime}+3 \kappa^{2} \kappa^{\prime}-6 \kappa \tau \tau^{\prime}\right)$,

$$
\begin{aligned}
\dot{\tau}= & \frac{\kappa^{\prime \prime \prime} \kappa-2 \kappa^{2} \tau \tau^{\prime}+\kappa^{3} \kappa^{\prime}-\kappa^{\prime} \kappa^{\prime \prime}}{\kappa^{2}} \\
& +\frac{\mu}{2 \kappa^{2}}\left[3 \kappa^{4} \tau^{\prime}+6 \kappa^{\prime \prime \prime} \kappa \tau+2 \kappa^{2} \tau^{\prime \prime \prime}+12 \kappa \kappa^{\prime \prime} \tau^{\prime}-6 \kappa^{2} \tau^{2} \tau^{\prime}\right. \\
& \left.+6 \kappa \kappa^{\prime} \tau^{\prime \prime}-6\left(\kappa^{\prime}\right)^{2} \tau^{\prime}+6 \kappa^{3} \tau \kappa^{\prime}-6 \tau \kappa^{\prime} \kappa^{\prime \prime}\right]
\end{aligned}
$$

If we take $\mu=0$ in Eqs. (15) and (16), the Da Rios equations are reproduced [59].

We consider linear fluctuations, $\delta \kappa$ and $\delta \tau$, around constant $\kappa$ and $\tau$. By taking $\delta \kappa, \delta \tau \propto e^{-i \omega t+i p s}$, the dispersion relation is obtained from Eqs. (15) and (16) as follows: 


$$
\begin{array}{r}
\omega= \\
2 p \tau+\mu p\left(p^{2}-\frac{3}{2} \kappa^{2}+3 \tau^{2}\right) \\
\pm \sqrt{p^{2}\left(p^{2}-\kappa^{2}\right)(1+3 \mu \tau)^{2}} .
\end{array}
$$

Equation (17) compactly encodes the information of the fluctuations around three different configurations: a circle, a helix, and a straight line. Let us first discuss a circle, in which case the torsion is zero. The periodicity of a circle requires $p=n \kappa$ with an integer $n$, then the frequency $\omega$ simplifies to

$$
\omega= \pm \kappa^{2} \sqrt{n^{2}\left(n^{2}-1\right)}+\mu \kappa^{3} n\left(n^{2}-\frac{3}{2}\right)
$$

If we take $\mu=0$, Eq. (18) coincides with the result of previous studies [60-63]. The mode with $n=0$ corresponds to the change of radius, $n= \pm 1$ represents a slight change of the propagation direction, and does not involve the change of its shape. At $\mu=0$, these modes are the zero modes of the soliton. At $\mu \neq 0$, because of the chirality imbalance, $n= \pm 1$ modes acquire finite frequencies. The degeneracy between $n \leftrightarrow-n$ is also lifted. The frequency is always real, which means that a circle is stable.

Let us now consider the helix-shape solution. The lowest value of $p$ is determined by the length $L$ of a helix as $2 \pi / L$. At $\mu=0$, the imaginary part appears if $p^{2}<\kappa^{2}$, which means that these long-wavelength modes are unstable. Because the factor $(1+3 \mu \tau)^{2}$ is always nonnegative, this condition is unchanged, except for a very special choice of the chiral chemical potential $\mu \tau=-1 / 3$. However, a finite $\mu$ changes the growth rate of unstable modes. In the small $p$ limit, the growth rate is given by

$$
\operatorname{Im} \omega= \pm \sqrt{\kappa^{2}(1+3 \mu \tau)^{2}} p+O\left(p^{2}\right),
$$

which is different for the right-handed $(\tau>0)$ and lefthanded $(\tau<0)$ helices. The real part of $\omega$ to the first order in $p$ is given by

$$
\operatorname{Re} \omega=\left(2 \tau+\frac{3}{2} \mu\left(2 \tau^{2}-\kappa^{2}\right)\right) p+O\left(p^{2}\right) .
$$

Hence, the chirality imbalance also modifies the velocity of the wave propagating along the helix.

In the limit $\kappa \rightarrow 0, \tau \rightarrow 0$, the helix approaches a straight line [64], and the dispersion for the fluctuations around a straight vortex, $\omega= \pm p^{2}+\mu p^{3}$, is obtained. The leading $p^{2}$ behavior corresponds to the famous Kelvin waves [65], and the second term represents the modification due to the chirality imbalance.

Absence of propagation of planar solutions in the PTsymmetric case.-In the case of the LIE, the velocity of a Hasimoto soliton is given by $v=2 \tau_{0}$ [take $\mu=0$ in

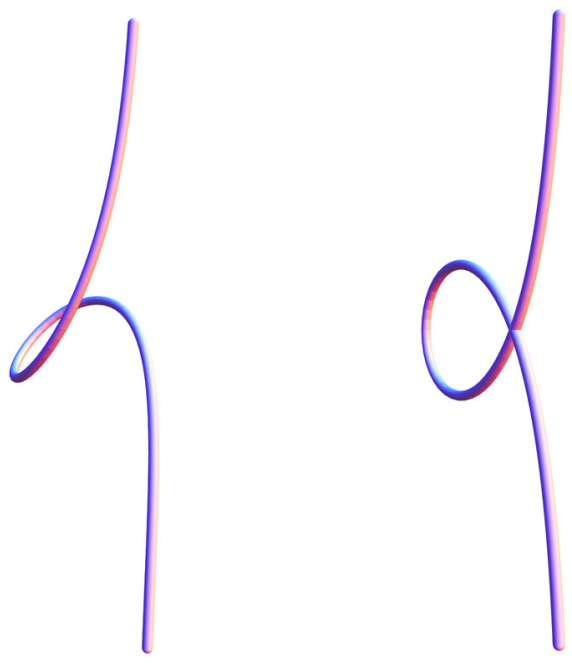

FIG. 1. Nonplanar [left, $\left(\epsilon, \tau_{0}, t\right)=(1,0.4,0)$ ] and planar [right, $\left(\epsilon, \tau_{0}, t\right)=(1,0,0)$ ] solitons.

Eq. (7)]. At $\tau \neq 0$, the solutions are chiral, in the sense that their handedness is correlated with the direction of the propagation. A mirror image of a solution propagates in the opposite direction from the original one. If we look at a planar $(\tau=0)$ solution at $\mu=0$ [see the right side of Fig. 1], it just rotates around its axis and cannot convey energy along the vortex. In fact, this is a generic feature of a planar solution in the LIE. Here, we consider a class of solutions that are asymptotically straight lines, like the Hasimoto solitons. We now show that planar solutions cannot propagate if the equation of motion (EOM) has the $P T$ symmetry. The LIE has this symmetry, whereas the FME does not.

It suffices to show that, when the solution is planar, the velocity $\dot{\boldsymbol{X}}$ is restricted to the direction of $\boldsymbol{b}$, because a binormal motion cannot make the soliton propagate along the vortex. Consider a current written in the form

$$
\boldsymbol{J}=\int d s f(\kappa, \tau) \dot{\boldsymbol{X}}
$$

where $f(\kappa, \tau)$ is a function of $\kappa$ and $\tau$. The energy current is written in this form. Let us denote the unit vector in the direction of the asymptotic line by $\boldsymbol{\ell}$. Because $\boldsymbol{\ell}$ is within the plane spanned by $\{\boldsymbol{t}, \boldsymbol{n}\}$ for a planar solution, it is always orthogonal to $\boldsymbol{b}, \boldsymbol{l} \cdot \boldsymbol{b}=0$. Thus, if $\dot{\boldsymbol{X}} \propto \boldsymbol{b}$, then $\boldsymbol{\ell}$. $\boldsymbol{J}=0$ holds and there is no energy flow in the direction of $\boldsymbol{\ell}$.

Let us examine the transformation property of the EOMs. The parity reflection, $\boldsymbol{X} \rightarrow-\boldsymbol{X}$, acts on the FS system as

$$
\{\boldsymbol{t}, \boldsymbol{n}, \boldsymbol{b}, \kappa, \tau\} \rightarrow\{-\boldsymbol{t},-\boldsymbol{n}, \boldsymbol{b}, \kappa,-\tau\} .
$$

The binormal vector is parity even, because $\boldsymbol{b}=\boldsymbol{t} \times \boldsymbol{n}$, while the torsion $\tau=\left(\boldsymbol{n}^{\prime} \cdot \boldsymbol{b}\right)$ is parity odd. The right hand 
side (RHS) of the LIE, $\boldsymbol{X}^{\prime} \times \boldsymbol{X}^{\prime \prime}$, is $P$ even, while the modification to the LIE in the FME, $\boldsymbol{X}^{\prime \prime \prime}+\frac{3}{2}\left(\boldsymbol{X}^{\prime \prime}\right)^{2} \boldsymbol{X}^{\prime}$, is $P$ odd.

A general EOM can be written in the form

$$
\dot{\boldsymbol{X}}=a(\kappa, \tau) \boldsymbol{t}+b(\kappa, \tau) \boldsymbol{n}+c(\kappa, \tau) \boldsymbol{b} .
$$

From the assumption, the theory has the $P T$ symmetry. The LHS is even under $P T$. The RHS is $T$ even, so it has to be $P$ even. For a planar solution, $\tau=0$, the coefficients in Eq. (23) are all $P$ even, because $\kappa$ is a $P$-even quantity. Thus, the coefficients of $\boldsymbol{t}$ and $\boldsymbol{n}$ have to vanish, $a(\kappa, \tau=0)=b(\kappa, \tau=0)=0$, and $\dot{\boldsymbol{X}} \propto \boldsymbol{b}$.

The results above can be further generalized. The LIE can be mapped to NLSE, which has an infinite sequence of commuting invariants. Those invariants are the generators of Hamiltonian flows. Correspondingly, the LIE also has infinitely many commuting Hamiltonian flows [47], which are called the LIE hierarchy. The first and second terms of the RHS of the FME (4) are the first two Hamiltonian flows

$$
\boldsymbol{V}_{0}=\kappa \boldsymbol{b}, \quad \boldsymbol{V}_{1}=\frac{\kappa^{2}}{2} \boldsymbol{t}+\kappa^{\prime} \boldsymbol{n}+\kappa \tau \boldsymbol{b}, \ldots
$$

In Ref. [47], a recursion operator that successively generates the next flow is constructed

$$
\mathcal{R} \boldsymbol{V} \equiv-\mathcal{P}\left[\boldsymbol{t} \times \partial_{s} \boldsymbol{V}\right]
$$

where $\mathcal{P}$ denotes the reparametrization procedure to keep the arc-length-preserving nature, which is done by adding a tangential term (see also Ref. [53]). Once we know $\boldsymbol{V}_{n}$, we can obtain the next flow by $\boldsymbol{V}_{n+1}=\mathcal{R} \boldsymbol{V}_{n}$. One can show [58] that $\boldsymbol{V}_{n}$ is $P$ even (odd) if $n$ is an even (odd) number. Thus, every EOM with an even $n$ has the $P T$ symmetry, and the solution of the EOM cannot propagate if its planar.

To summarize, we have found a new phenomenon affecting the dynamics of vortex solitons in chirally imbalanced media-the chiral propulsion effect. The CPE refers to an additional energy flow along the vortex filament in the direction determined by chirality imbalance. The energy is carried along the vortex by helical excitations analogous to the Hasimoto solitons. We have also found that a chirality imbalance modifies the excitation spectrum around solutions, one example of which is the modification of the growth rate of instability around a helical soliton solution. It is shown that, if the EOM respects the $P T$ symmetry, a planar solution cannot transfer energy-this indicates that the existence of the CPE is attributed to the breaking of parity in the medium. Because the total momentum of the system is conserved, the momentum transfer to the helical solitons will be balanced by the rest of the medium. The phenomena addressed in this Letter may be realized in chiral fluids in the presence of nonzero magnetic helicity-for example, Weyl and Dirac semimetals in external parallel electric and magnetic fields, or a quark-gluon plasma in heavy-ion collisions.

This work was supported in part by the U.S. Department of Energy under Contracts No. DE-FG-88ER40388 and No. DE-SC-0017662 (D. E. K.), No. DE-AC0298CH10886 (Y. H. and D. E. K.), and within the framework of the Beam Energy Scan Theory Topical Collaboration. The work of A. V.S. is partially supported through the LANL LDRD Program. A. V. S is grateful for support from RFBR Grant No. 17-02-01108 at the beginning of this work. The work of Y. H. was supported in part by the Korean Ministry of Education, Science and Technology, Gyeongsangbuk-do and Pohang City for Independent Junior Research Groups at the Asia Pacific Center for Theoretical Physics.

[1] S. L. Adler, Phys. Rev. 177, 2426 (1969).

[2] J.S. Bell and R. Jackiw, Il Nuovo Cimento A 60, 47 (1969).

[3] D. Kharzeev, Phys. Lett. B 633, 260 (2006).

[4] D. E. Kharzeev, L. D. McLerran, and H. J. Warringa, Nucl. Phys. A803, 227 (2008).

[5] K. Fukushima, D. E. Kharzeev, and H. J. Warringa, Phys. Rev. D 78, 074033 (2008).

[6] D. E. Kharzeev, J. Liao, S. A. Voloshin, and G. Wang, Prog. Part. Nucl. Phys. 88, 1 (2016).

[7] D. Kharzeev, K. Landsteiner, A. Schmitt, and H.-U. Yee, Lect. Notes Phys. 871, 1 (2013).

[8] D. Kharzeev and A. Zhitnitsky, Nucl. Phys. A797, 67 (2007).

[9] J. Erdmenger, M. Haack, M. Kaminski, and A. Yarom, J. High Energy Phys. 01 (2009) 055.

[10] N. Banerjee, J. Bhattacharya, S. Bhattacharyya, S. Dutta, R. Loganayagam, and P. Surowka, J. High Energy Phys. 01 (2011) 094.

[11] M. Torabian and H.-U. Yee, J. High Energy Phys. 08 (2009) 020.

[12] D. T. Son and P. Surowka, Phys. Rev. Lett. 103, 191601 (2009).

[13] Q. Li, D. E. Kharzeev, C. Zhang, Y. Huang, I. Pletikosic, A. V. Fedorov, R. D. Zhong, J. A. Schneeloch, G. D. Gu, and T. Valla, Nat. Phys. 12, 550 (2016).

[14] M. Joyce and M. E. Shaposhnikov, Phys. Rev. Lett. 79, 1193 (1997).

[15] Y. Akamatsu and N. Yamamoto, Phys. Rev. Lett. 111, 052002 (2013).

[16] A. Avdoshkin, V. P. Kirilin, A. V. Sadofyev, and V. I. Zakharov, Phys. Lett. B 755, 1 (2016).

[17] H. Tashiro, T. Vachaspati, and A. Vilenkin, Phys. Rev. D 86, 105033 (2012).

[18] Y. Hirono, D. E. Kharzeev, and Y. Yin, Phys. Rev. D 92, 125031 (2015).

[19] N. Yamamoto, Phys. Rev. D 93, 125016 (2016).

[20] K. Hattori, Y. Hirono, H.-U. Yee, and Y. Yin, arXiv: 1711.08450 . 
[21] J. Xiong, S. K. Kushwaha, T. Liang, J. W. Krizan, M. Hirschberger, W. Wang, R. Cava, and N. Ong, Science 350, 413 (2015).

[22] C.-Z. Li, L.-X. Wang, H. Liu, J. Wang, Z.-M. Liao, and D.-P. Yu, Nat. Commun. 6, 10137 (2015).

[23] C. Zhang, S.-Y. Xu, I. Belopolski, Z. Yuan, Z. Lin, B. Tong, N. Alidoust, C.-C. Lee, S.-M. Huang, H. Lin et al., arXiv: 1503.02630.

[24] X. Yang, Y. Liu, Z. Wang, Y. Zheng, and Z.-a. Xu, arXiv: 1506.03190

[25] X. Yang, Y. Li, Z. Wang, Y. Zhen, and Z.-a. Xu, arXiv: 1506.02283 .

[26] V. Koch, S. Schlichting, V. Skokov, P. Sorensen, J. Thomas, S. Voloshin, G. Wang, and H.-U. Yee, Chin. Phys. C 41, 072001 (2017).

[27] L. Adamczyk et al. (STAR Collaboration), Nature 548, 62 (2017).

[28] Z.-T. Liang and X.-N. Wang, Phys. Rev. Lett. 94, 102301 (2005); 96, 039901(E) (2006).

[29] B. Betz, M. Gyulassy, and G. Torrieri, Phys. Rev. C 76, 044901 (2007).

[30] F. Becattini, L. P. Csernai, and D. J. Wang, Phys. Rev. C 88, 034905 (2013); 93, 069901(E) (2016).

[31] F. Becattini, G. Inghirami, V. Rolando, A. Beraudo, L. Del Zanna, A. De Pace, M. Nardi, G. Pagliara, and V. Chandra, Eur. Phys. J. C 75, 406 (2015); 78, 354(E) (2018).

[32] M. I. Baznat, K. K. Gudima, A. S. Sorin, and O. V. Teryaev, Phys. Rev. C 93, 031902 (2016).

[33] L.-G. Pang, H. Petersen, Q. Wang, and X.-N. Wang, Phys. Rev. Lett. 117, 192301 (2016).

[34] W.-T. Deng and X.-G. Huang, Phys. Rev. C 93, 064907 (2016).

[35] Y. Jiang, Z.-W. Lin, and J. Liao, Phys. Rev. C 94, 044910 (2016).

[36] F. Becattini, I. Karpenko, M. A. Lisa, I. Upsal, and S. A. Voloshin, Phys. Rev. C 95, 054902 (2017).

[37] I. Karpenko and F. Becattini, Eur. Phys. J. C 77, 213 (2017).

[38] H. Li, H. Petersen, L.-G. Pang, Q. Wang, X.-L. Xia, and X.-N. Wang, Nucl. Phys. A967, 772 (2017).
[39] A. Aristova, D. Frenklakh, A. Gorsky, and D. Kharzeev, J. High Energy Phys. 10 (2016) 029.

[40] R. Arms and F. R. Hama, Phys. Fluids 8, 553 (1965).

[41] R. L. Ricca, Nature 352, 561 (1991).

[42] P. G. Saffman, Vortex Dynamics (Cambridge University Press, Cambridge, England, 1992).

[43] A. Vilenkin and E.P.S. Shellard, Cosmic Strings and Other Topological Defects (Cambridge University Press, Cambridge, England, 2000).

[44] G. E. Volovik, The Universe in a Helium Droplet (Oxford University Press, New York, 2009), Vol. 117.

[45] M. Eto, Y. Hirono, M. Nitta, and S. Yasui, Prog. Theor. Exp. Phys. 2014, 012D01 (2014).

[46] H. Hasimoto, J. Fluid Mech. 51, 477 (1972).

[47] J. Langer and R. Perline, J. Nonlinear Sci. 1, 71 (1991).

[48] E. Hopfinger and F. Browand, Nature 295, 393 (1982).

[49] E. Hopfinger, F. Browand, and Y. Gagne, J. Fluid Mech. 125, 505 (1982).

[50] D. E. Kharzeev, Prog. Part. Nucl. Phys. 75, 133 (2014).

[51] A. A. Kozhevnikov, Phys. Lett. B 461, 256 (1999).

[52] A. A. Kozhevnikov, Phys. Lett. B 750, 122 (2015).

[53] D. D. Holm and S. N. Stechmann, arXiv:nlin/0409040.

[54] Y. Fukumoto and T. Miyazaki, J. Fluid Mech. 222, 369 (1991).

[55] T. Kambe, Geometrical Theory of Dynamical Systems and Fluid Flows (World Scientific, Singapore, 2004), Vol. 23.

[56] R. Hirota, J. Math. Phys. (N.Y.) 14, 805 (1973).

[57] R. L. Ricca, Phys. Fluids A 4, 938 (1992).

[58] See Supplemental Material at http://link.aps.org/ supplemental/10.1103/PhysRevLett.121.142301 for details.

[59] L. Da Rios, Rend. Circ. Mat. Palermo 22, 117 (1906).

[60] T. Kambe and T. Takao, J. Phys. Soc. Jpn. 31, 591 (1971).

[61] R. Betchov, J. Fluid Mech. 22, 471 (1965).

[62] R. L. Ricca, Fluid Dyn. Res. 18, 245 (1996).

[63] K. Suzuki, T. Ono, and T. Kambe, Phys. Rev. Lett. 77, 1679 (1996).

[64] R. L. Ricca, J. Fluid Mech. 273, 241 (1994).

[65] W. Thomson, Lond. Edinburgh Dublin Philos. Mag. J. Sci. 10, 155 (1880). 